\title{
Adult invasive pneumococcal disease pre- and peri-pneumococcal conjugate vaccine introduction in a tertiary hospital in Singapore
}

\begin{abstract}
Correspondence
Li-Yang Hsu

liyang_hsu@yahoo.com
\end{abstract}

Received 27 May 2008

Accepted 4 September 2008
Li-Yang Hsu, ${ }^{1}$ See Weng Lui, ${ }^{2}$ Jia Ling Lee, ${ }^{2}$ Hazwany Md Mahrom Hedzlyn, ${ }^{2}$ Debra Han-Lin Kong, ${ }^{2}$ Suku Shameen, ${ }^{2}$ Nur Puteri Mohammad Siti, ${ }^{2}$ Wei Yu Tan, ${ }^{2}$ Xin Yi Toh, ${ }^{2}$ Tse-Yuen Koh ${ }^{2}$ and Tse-Hsien Koh ${ }^{3}$

\author{
${ }^{1}$ Yong Loo Lin School of Medicine, National University of Singapore, Singapore \\ ${ }^{2}$ School of Life Sciences \& Chemical Technology, Ngee Ann Polytechnic, Singapore \\ ${ }^{3}$ Singapore General Hospital, Singapore
}

\begin{abstract}
Streptococcus pneumoniae isolates causing invasive disease at a large tertiary institute in Singapore from 2000 to 2007 were serotyped, with 84 (43.8\%) and $159(82.8 \%)$ isolates belonging to serotypes covered by the pneumococcal heptavalent conjugate and polysaccharide vaccines, respectively. All non-meningitis isolates were susceptible to penicillin, and the attributable mortality was $21.4 \%$. Patients who fulfilled the US Advisory Committee on Immunization Practices criteria for vaccination with the pneumococcal polysaccharide vaccine comprised $74.0 \%$ of the study cohort and had a significantly higher mortality risk.
\end{abstract}

\section{INTRODUCTION}

Pneumococcal infections remain a major cause of morbidity and mortality worldwide, despite the widespread availability of two vaccines: the 23-valent polysaccharide vaccine (PPV) and the heptavalent conjugate vaccine (PCV7). This problem has been exacerbated by the global increase in antimicrobial resistance in Streptococcus pneumoniae (Adam, 2002), a phenomenon that is particularly striking in Asia (Song et al., 2004). Nonetheless, the early impact of PCV7 appears significant - a substantial drop in pneumococcal disease incidence in both adults and children has been reported following the incorporation of this vaccine into the US childhood immunization programme, despite clear evidence of serotype replacement (Black et al., 2006; Hicks et al., 2007).

In Singapore, PPV and PCV7 were licensed for local use in 1988 and 2002, respectively (Low et al., 2007), although the latter was only officially launched in October 2005 and has not been incorporated into the country's national childhood immunization programme. There is no concerted surveillance programme for S. pneumoniae locally, and sporadic serotyping work to date has focused mainly on paediatric isolates (Soh et al., 2000). In this study, we archived all invasive pneumococcal isolates cultured from sterile sites from adult patients hospitalized at Singapore

Abbreviations: ACIP, Advisory Committee on Immunization Practices; PCV7, heptavalent conjugate vaccine; PPV, 23-valent polysaccharide vaccine.
General Hospital - a 1600-bed tertiary hospital that provides almost a quarter of all public-sector acute hospital beds in the country - since 2000 , and sought to determine both clinical characteristics and microbiological trends, including serotype distribution, in the period pre- and peri-PCV7 introduction.

\section{METHODS}

Isolates were confirmed as $S$. pneumoniae by colony morphology, $\alpha$ haemolysis and optochin susceptibility on blood agar. Serotyping was performed using a combination of multiplex PCR (Pai et al., 2006), latex agglutination using a Pneumotest-Latex kit (Statens Serum Institut) and Quellung reaction with factor antisera (Statens Serum Institut). The MICs of isolates to penicillin were evaluated using Etest (AB Biodisk). Strain typing and MIC testing experiments were performed twice, with identical results each time.

Demographic and clinical data were obtained by a review of the patients' medical records, and an investigator blind to the serotyping and penicillin MIC results determined whether mortality was attributable to pneumococcal infection. Intercooled Stata (version 10.0) was used for statistical calculations.

\section{RESULTS AND DISCUSSION}

Pneumococcal isolates from 192 patients were archived between 1 January 2000 and 31 December 2007. The majority $(92.7 \%)$ were from blood cultures, with the remainder obtained from pleural fluid $(2.1 \%)$, intraophthalmic (1.6\%), CSF (1.0\%), synovium (1.0\%), 
Table 1. Demographic and clinical characteristics of patients with invasive pneumococcal disease presenting at Singapore General Hospital from 2001 to 2007, and serotypes of the causative isolates

\begin{tabular}{|c|c|}
\hline Characteristic & No. of patients \\
\hline \multicolumn{2}{|l|}{ Demographics } \\
\hline Male gender (\%) & $141(73.4)$ \\
\hline Age, median years (range) & $64(14-96)$ \\
\hline Chinese ethnicity (\%) & $137(71.4)$ \\
\hline Malay ethnicity (\%) & $37(19.3)$ \\
\hline Indian ethnicity (\%) & $11(5.7)$ \\
\hline $\begin{array}{l}\text { Fulfilling ACIP (1997) criteria for PPV } \\
\text { vaccination }(\%)\end{array}$ & $142(74.0)$ \\
\hline \multicolumn{2}{|l|}{ Type of infection (\%) } \\
\hline Pneumonia & $180(93.8)$ \\
\hline Bacteraemia & $182(94.8)$ \\
\hline Empyema of the lung & $7(3.6)$ \\
\hline Meningitis & $6(3.1)$ \\
\hline Septic arthritis & $5(2.6)$ \\
\hline Endocarditis & $4(2.1)$ \\
\hline Endophthalmitis & $3(1.6)$ \\
\hline Sinusitis & $3(1.6)$ \\
\hline Pericarditis & $2(1.0)$ \\
\hline Infected aortic aneurysm & $2(1.0)$ \\
\hline \multicolumn{2}{|l|}{ Empirical antibiotic therapy (\%) } \\
\hline Ceftriaxone monotherapy & $76(39.6)$ \\
\hline Ceftriaxone + macrolide & $53(27.6)$ \\
\hline $\begin{array}{l}\text { Respiratory fluoroquinolone alone or in } \\
\text { combination* }\end{array}$ & $9(4.7)$ \\
\hline Cefepime monotherapy & $7(3.6)$ \\
\hline Piperacillin/tazobactam & $5(2.6)$ \\
\hline Vancomycin & $5(2.6)$ \\
\hline Ciprofloxacin & $4(2.1)$ \\
\hline Ceftazidime + macrolide & $4(2.1)$ \\
\hline Amoxicillin/clavulanate + macrolide & $3(1.6)$ \\
\hline Penicillin monotherapy & $3(1.6)$ \\
\hline Other & $22(11.5)$ \\
\hline No empiric antibiotics initiated & $1(0.5)$ \\
\hline \multicolumn{2}{|l|}{ Pneumococcal serotype (\%) } \\
\hline All & $192(100)$ \\
\hline 1 & $8(4.2)$ \\
\hline 3 & $22(11.5)$ \\
\hline 4 & $7(3.6)$ \\
\hline $6 \mathrm{~A}$ & $4(2.1)$ \\
\hline $6 \mathrm{~B}$ & $16(8.3)$ \\
\hline 8 & $13(6.8)$ \\
\hline $12 \mathrm{~F}$ & $6(3.1)$ \\
\hline 14 & $35(18.2)$ \\
\hline $15 \mathrm{~A} / \mathrm{C} / \mathrm{F}$ & $9(4.7)$ \\
\hline $19 \mathrm{~A}$ & $4(2.1)$ \\
\hline $19 \mathrm{~F}$ & $12(6.3)$ \\
\hline 20 & $9(4.7)$ \\
\hline $22 \mathrm{~F}$ & $4(2.1)$ \\
\hline $23 \mathrm{~F}$ & $8(4.2)$ \\
\hline $33 \mathrm{~F}$ & $5(2.6)$ \\
\hline Other serotypes $\dagger$ & $30(15.6)$ \\
\hline
\end{tabular}

endothelial $(1.0 \%)$ and lung tissue $(0.5 \%)$ cultures. The demographic and clinical characteristics of these patients are shown in Table 1, along with the serotypes of the isolates.

The ethnic distribution of the patients broadly reflected the national ethnic distribution (Singapore Department of Statistics, 2007). The majority were male and met at least one of the Advisory Committee on Immunization Practices (ACIP) criteria for PPV vaccination (ACIP, 2007). Most of the patients had pneumonia with concomitant bacteraemia, and none had received pneumococcal vaccination previously. None had received antibiotic therapy in the month preceding their infection. The median penicillin MIC was $0.016 \mu \mathrm{g} \mathrm{ml}^{-1}$ (range $0.016-2 \mu \mathrm{g} \mathrm{ml}^{-1}$ ), as was the median ceftriaxone MIC (range $0.004-1 \mu \mathrm{g} \mathrm{ml}^{-1}$ ). All 186 non-meningitis isolates would be classified as penicillin-susceptible following the new CLSI breakpoints (CLSI, 2008); one isolate from a patient with meningitis had an MIC to penicillin of $2 \mu \mathrm{g} \mathrm{ml}^{-1}$, but the other five isolates were susceptible.

Five patients $(2.6 \%)$ did not receive an empiric antibiotic with significant anti-pneumococcal coverage, and one $(0.5 \%)$ did not receive any empiric antibiotic. Forty-one patients $(21.4 \%)$ died from pneumococcal septicaemia, including both patients (100\%) with infected aortic aneurysms, one patient $(25 \%)$ with endocarditis and one patient $(16.6 \%)$ with meningitis. On univariate analysis, patients who fulfilled the ACIP criteria were at higher risk of mortality [odds ratio (OR) 8.14; $95 \%$ CI 1.88-35.21; $P=0.005$ ]. Male gender (OR 1.64; $P=0.25$ ), ethnicity (OR $0.89 ; P=0.63)$, infection by a $P C V 7$-associated serotype (OR 1.41; $P=0.34$ ) and use of appropriate empiric antibiotics (OR $0.34 ; P=0.17$ ) were not associated with mortality.

Serotypes 3, 6B and 14 were the most common, comprising $38.0 \%$ of all isolates. Serotypes covered by PCV7 and PPV comprised 43.8 and $82.8 \%$ of all isolates, respectively. The distribution of isolates according to vaccine coverage and fulfilment of ACIP PPV criteria is shown in Fig. 1. Because the numbers were small, it was not possible to demonstrate a significant shift in any individual serotype over this period. However, the proportion and numbers of PCV7associated serotypes did not decrease significantly in the 2 years after introduction of the vaccine $(P=0.30)$, and the proportion of serotypes covered by both vaccines did not alter over the 8 years $(P=0.74)$.

\footnotetext{
${ }^{*}$ Includes moxifloxacin or levofloxacin. These were combined with ceftazidime (three cases), ceftazidime + cloxacillin (three cases), clindamycin (one case) or used as monotherapy (two cases).

$\dagger$ There was one isolate each of serogroups 2, 9AL, 10A, 15B, 22A, $33 \mathrm{~A} / \mathrm{B} / \mathrm{C} / \mathrm{D}, 35 \mathrm{~F}$ and 38 , two isolates each of serogroups $5,7 \mathrm{~F}, 17 \mathrm{~A}$, $18 \mathrm{~A} / \mathrm{B}$ and $19 \mathrm{~B} / \mathrm{C}$, and three isolates each of serogroups $7 \mathrm{C}$ and 18C. There were also six isolates classified as belonging to non-vaccine serotypes using the Pneumotest-Latex kit and one isolate that could not be serotyped based on the three methods employed.
} 


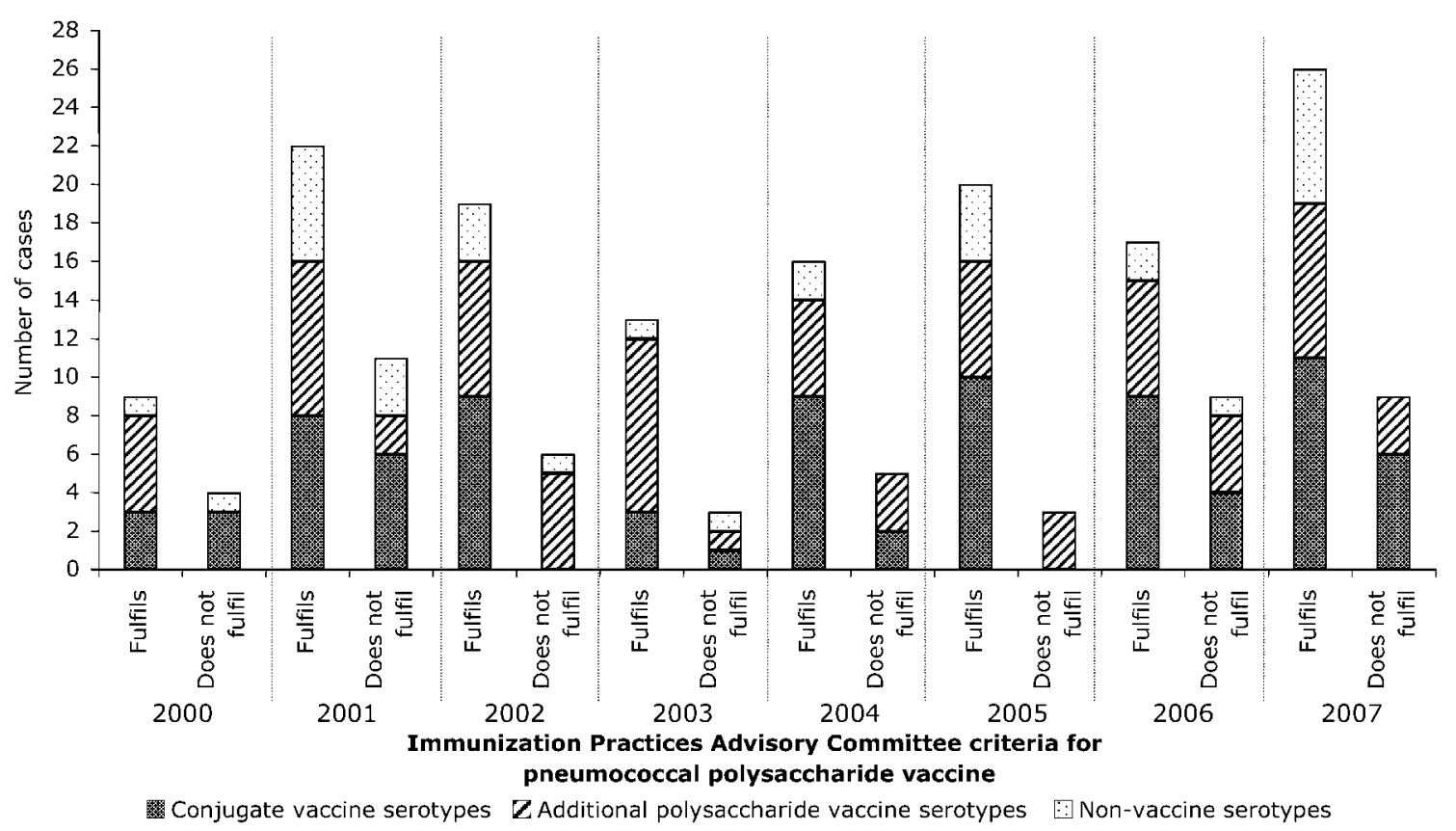

Fig. 1. Distribution of invasive S. pneumoniae isolates according to vaccine coverage and fulfilment of the ACIP polysaccharide pneumococcal vaccine criteria.

Our results suggest that PCV7 has had little impact on adult invasive pneumococcal disease in Singapore. This is unsurprising given its recent introduction and exclusion from the national childhood immunization programme. It is noteworthy that the proportion of invasive pneumococcal disease occurring in patients fulfilling the ACIP criteria for PPV vaccination has not fallen in the past 8 years, but lack of relevant denominator data prevents further valid commentary.

Although previous reports have highlighted a rising incidence of penicillin non-susceptibility among local pneumococcal isolates (Song et al., 2004), the recent change in CLSI breakpoints resulted in virtually all of our isolates testing as susceptible to penicillin (CLSI, 2008). Applying these breakpoints to previous studies (Soh et al., 2000; Song et al., 2004; Chong et al., 2006) results in similar conclusions. Nonetheless, the mortality from invasive pneumococcal infections is very high, despite appropriate antibiotics being prescribed in the vast majority (96.9\%) of patients, with the risk of dying highest in patients who fulfil the ACIP criteria for PPV vaccination - these patients formed almost three-quarters of our patient cohort. This is similar to findings from other centres (Plouffe et al., 1996; Balakrishnan et al., 2000).

In conclusion, whilst coverage of invasive pneumococcal isolates by either PCV7 or PPV is by no means complete, the high prevalence $(74.0 \%)$ of patients who fulfil the ACIP criteria for PPV vaccination in our study suggests that appropriate use of PPV may result in a decrease in preventable deaths caused by $S$. pneumoniae among adults in Singapore. There is also evidence to suggest that routine immunization of children with PCV7 may also decrease the incidence of invasive pneumococcal disease among adults (CDC, 2005; Albrich et al., 2007). Because of observations that some degree of serotype replacement by non-vaccine serotypes may occur in the face of vaccine pressure, it is necessary to continue regular surveillance of the serotypes of pneumococci causing disease (Black et al., 2006; Hicks et al., 2007).

\section{ACKNOWLEDGEMENTS}

This study was funded by Merck Sharp and Dohme, and student project grants from Ngee Ann Polytechnic, Singapore.

\section{REFERENCES}

Adam, D. (2002). Global antibiotic resistance in Streptococcus pneumoniae. J Antimicrob Chemother 50 (Suppl.), 1-5.

ACIP (1997). Prevention of pneumococcal disease: recommendations of the Advisory Committee on Immunization Practices (ACIP). MMWR Recomm Rep 46 (RR-8), 1-24.

Albrich, W. C., Baughman, W., Schmotzer, B. \& Farley, M. M. (2007). Changing characteristics of invasive pneumococcal disease in Metropolitan Atlanta, Georgia, after introduction of a 7-valent pneumococcal conjugate vaccine. Clin Infect Dis 44, 1569-1576.

Balakrishnan, I., Crook, P., Morris, R. \& Gillespie, S. H. (2000). Early predictors of mortality in pneumococcal bacteraemia. J Infect 40, 256-261.

Black, S., Shinefield, H., Baxter, R., Austrian, R., Elvin, L., Hansen, J., Lewis, E. \& Fireman, B. (2006). Impact of the use of heptavalent 
pneumococcal conjugate vaccine on disease epidemiology in children and adults. Vaccine 24 (Suppl. 2), S79-S80.

CDC (2005). Direct and indirect effects of routine vaccination of children with 7-valent pneumococcal conjugate vaccine on incidence of invasive pneumococcal disease - United States, 1998-2003. MMWR Morb Mortal Wkly Rep 54, 893-897.

Chong, C. Y., Mok, Y. H. \& Tee, N. (2006). Invasive pneumococcal disease in Singapore children. Poster presented at the 5th International Symposium on Pneumococci and Pneumococcal Diseases (ISPPDS), Alice Springs, Australia.

CLSI (2008). Performance Standards for Antimicrobial Susceptibility Testing. Eighteenth Informational Supplement, M100-S18. Wayne, PA: Clinical and Laboratory Standards Institute.

Hicks, L. A., Harrison, L. H., Flannery, B., Hadler, J. L., Schaffner, W., Craig, A. S., Jackson, D., Thomas, A., Beall, B. \& other authors (2007). Incidence of pneumococcal disease due to non-pneumococcal conjugate vaccine (PCV7) serotypes in the United States during the era of widespread PCV7 vaccination. J Infect Dis 196, 1346-1354.

Low, S., Chan, F. L. F., Cutter, J., Ma, S., Goh, K. T. \& Chew, S. K. (2007). A national study of the epidemiology of pneumococcal disease among hospitalised patients in Singapore: 1995 to 2004. Singapore Med J 48, 824-829.

Pai, R., Gertz, R. E. \& Beall, B. (2006). Sequential multiplex PCR approach for determining capsular serotypes of Streptococcus pneumoniae isolates. J Clin Microbiol 44, 124-131.

Plouffe, J. F., Breiman, R. F., Facklam, R. R. \& Franklin County Pneumonia Study Group (1996). Bacteremia with Streptococcus pneumoniae. Implications for therapy and prevention. JAMA 275, 194-198.

Singapore Department of Statistics (2007). Online Yearbook of Statistics Singapore 2007, http://www.singstat.gov.sg/pubn/reference/ yos/statsT-demography.pdf (accessed on 1 May 2008).

Soh, S. W., Poh, C. L. \& Lin, R. V. (2000). Serotype distribution and antimicrobial resistance of Streptococcus pneumoniae isolates from pediatric patients in Singapore. Antimicrob Agents Chemother 44, 2193-2196.

Song, J. H., Jung, S. I., Ko, K. S., Kim, N. Y., Son, J. S., Chang, H. H., Ki, H. K., Oh, W. S., Suh, J. Y. \& other authors (2004). High prevalence of antimicrobial resistance among clinical Streptococcus pneumoniae isolates in Asia (an ANSORP study). Antimicrob Agents Chemother 48, 2101-2107. 